\title{
A case of myocardial bridging: evaluation using intracoronary ultrasound, Doppler flow measurement, and quantitative coronary angiography
}

\author{
B J Kneale, A J Stewart, D J Coltart
}

Cardiac Department, St Thomas' Hospital,

London

B J Kneale

A J Stewart

D J Coltart

Correspondence to:

Dr D J Coltart, Cardiac

Department, St Thomas'

Hospital, Lambeth Palace

Road, London SE1 7EH.

Accepted for publication

25 April 1996

Figure 1 Selective coronary angiogram of the left coronary artery demonstrating systolic compression of a segment of the $L A D$ : the $L A D$ measures $2.5 \mathrm{~mm}$ in diameter (at $A$ and $B$ ) in diastole.

A 52 year old man was referred for investigation of chest pain. $\mathrm{He}$ had been treated at another hospital for unstable angina four months previously and subsequently underwent exercise stress testing. He performed six minutes of a Bruce protocol and developed angina with significant ST depression. At the time of referral he complained of chest pain on moderate exertion (Canadian Cardiovascular Society grade 2); he was an exsmoker and had a past history of hypertension. He was treated with aspirin $(75 \mathrm{mg})$, nifedipine (10 mg twice a day), and isosorbide mononitrate (20 mg twice a day).

Cardiac catheterisation was performed. The left ventriculogram showed inferior akinesia, anterior hypokinesia, and a left ventricular ejection fraction of $54 \%$. There was a total occlusion of the right coronary artery in midcourse. The circumflex coronary artery was blocked after $2 \mathrm{~cm}$. In the mid-course of the left anterior descending artery (LAD) there was systolic compression of the vessel but no evidence of atheromatous narrowing (figs 1 and 2). Further investigation was undertaken in order to determine the haemodynamic significance of this myocardial bridge.

An intravascular ultrasound study showed systolic compression of the LAD at the site of narrowing seen at concomitant angiography. Atheromatous plaque was noted proximal to the area of myocardial bridging but not distal to this region. Doppler flow velocimetry was performed which demonstrated a reduction of 
Figure 2 Selective coronary angiogram of the left coronary artery. In systole there is compression of $a$ $15 \mathrm{~mm}$ long segment of the $L A D$ to $1.25 \mathrm{~mm}$ in diameter (B), reference diameter (A) $2.5 \mathrm{~mm}$.
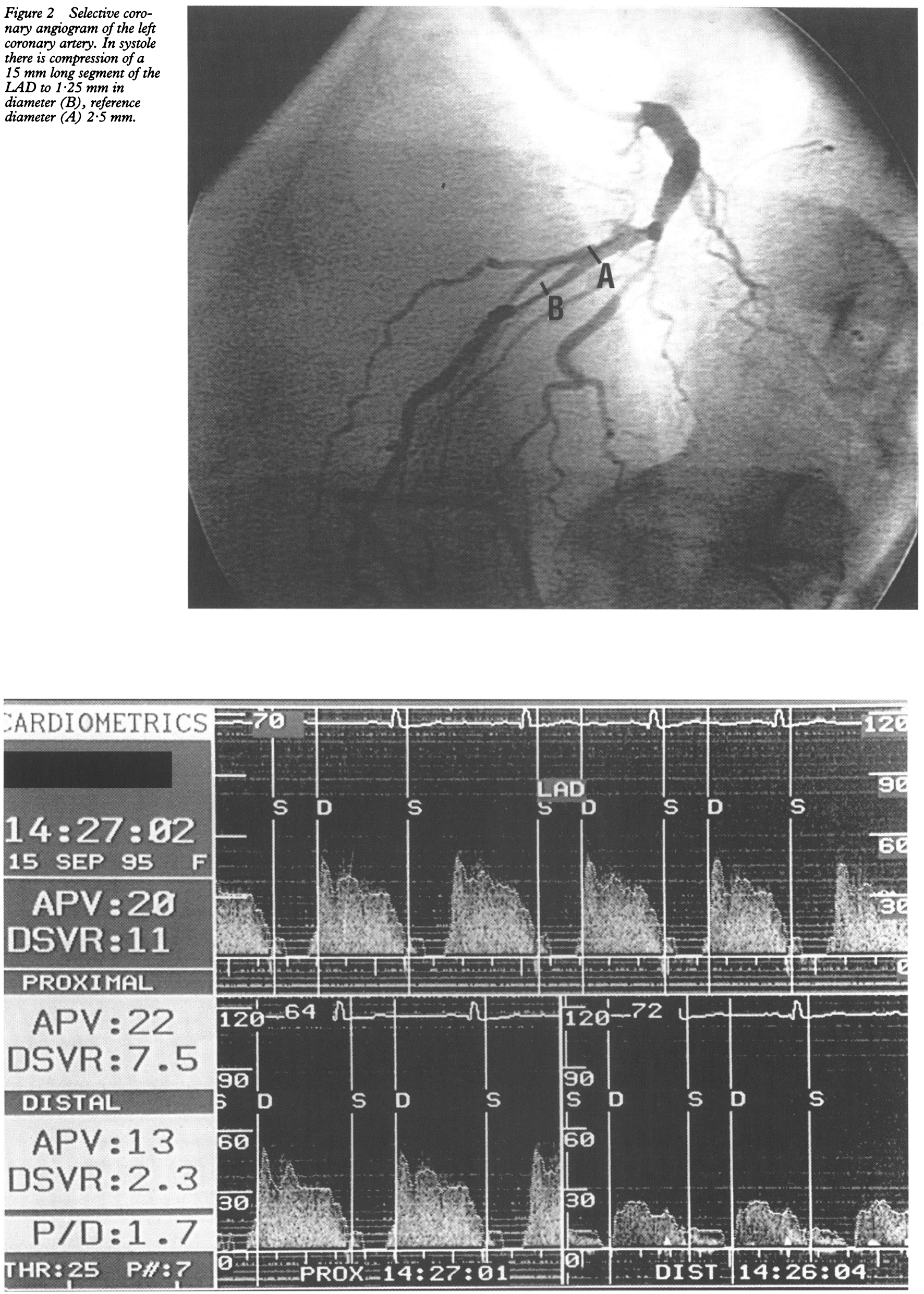

Figure 3 Tracing obtained from Doppler flow velocimetry within the LAD showing flow velocity profiles in segments proximal and distal to the segment of systolic compression. The average peak velocity (APV) is reduced distal to the myocardial bridge; however, more striking is the virtual absence of systolic flow on either side of the bridge (DSVR, diastolic:systolic velocity ratio). 
diastolic peak velocity distal to the myocardial bridge and virtually no systolic flow on either side of the lesion (fig 3). He was referred for coronary bypass surgery with the recommendation that the LAD be grafted.

Myocardial bridging is a well recognised phenomenon with a reported frequency of between $1 \cdot 6^{1}$ and $5 \cdot 5 \%^{2}$ at coronary angiography. It was found in $56 \%$ of 90 successive patients in a necropsy study. ${ }^{3}$ The marked discrepancy between the number of myocardial bridges demonstrable macroscopically at necropsy and the number of angiographically noted segments has been explained by distinguishing between two types of muscle bridge: a superficial type which does not cause constriction of the coronary artery and a deep type in which muscle fibres surround the LAD and may distort and compress the artery. ${ }^{3}$

In our patient we demonstrated a difference in coronary blood flow dynamics across the area of myocardial bridging and confirmed it by both quantitative coronary angiography and intravascular ultrasound. The presence of atheromatous plaque proximal to the area of compression but not distal to the bridge is an interesting feature. This was also reported in a study by $\mathrm{Ge}$ et al in which in 14 patients with myocardial bridging of the LAD, 12 had atheromatous plaque in the segment immediately proximal to the area of compression whereas in the eight patients in whom intracoronary ultrasound could be performed distal to the bridge there was no evidence of distal atheroma. ${ }^{4}$ The reason for the lack of distal atheroma is unclear but it may be because there is no systolic pressure wave (as we found in our patient by Doppler flow velocimetry) to injure the coronary endothelium in this position.

1 Ishimori T, Raizner AE, Chahine RJ, Awdeh M, Luchi RJ. Myocardial bridges in man: clinical correlations and angiographic accentuation with nitroglycerin. Cathet Cardiovasc Diagn 1977;3:59-65.

2 Angelini P, Trivellato M, Donis J, Leachman RD. Myocardial bridges: a review. Prog Cardiovasc Dis 1983;26: 75-88.

3 Ferreira Jr AG, Trotter SE, König Jr B, Décourt, Fox K, Olsen EGJ. Myocardial bridges: morphological and funcional aspects. Br Heart f 1991;66:364-7.

4 Ge J, Erbel R, Rupprecht H-J, Koch L, Kearney P, Görge $\mathrm{G}$, et al. Comparison of intravascular ultrasound and angiography in the assessment of myocardial bridging. Circulation 1994;89:1725-32. 